\title{
Hormonal Balance of Hyperthyroidism in the Laboratory of Biophysics of Dakar: Proposal of a Diagnostic and Monitoring Approach
}

\author{
Djiby Sow ${ }^{1,2,3}$, Amadou Ndiaye ${ }^{2 *}$, Boubacar Ndong ${ }^{4}$, Moussa Badiane ${ }^{4}$, \\ Pr Oumar Ndoye ${ }^{4}$ and Pr Niama Diop Sall ${ }^{2,3}$
}
${ }^{1}$ Marc Sankhale Center, Abass NDAO Hospital in Dakar, internal medicine service (Senegal)
${ }^{2}$ Laboratory analyzes Medical Biology, Abass NDAO hospital in Dakar (Senegal)
${ }^{3}$ Faculty of Medicine, Pharmacy and Dentistry (FMPOS) in Dakar, Medical Biochemistry Laboratory
${ }^{4}$ Faculty of Medicine, Pharmacy and Dentistry (FMPOS) in Dakar, biophysical laboratory

*Corresponding author

\section{A B S T R A C T}

\begin{tabular}{|l|}
\hline K e y w o r d s \\
Assessment, \\
Hyperthyroidism, \\
Diagnosis, \\
Surveillance
\end{tabular}

\section{Key words}

Assessment, Hyperthyroidism, Diagnosis, Surveillance

10 January 2018
Hyperthyroidism refers to a hyper functioning of the thyroid gland which increases the production of thyroid hormones because of thyrotoxicosis. Hyperthyroidism is a common endocrinopathy with multiple etiologies that can be its exploration requires a diagnostic approach consistent with the rational use of available biological means of exploration. Our aim was to determine the nature and prevalence of the principles underlying thyroid hormone demand in the investigation of clinically suspected or proven hyperthyroidism; to correlate the results of the hormonal exploration to the reasons. This study was carried out over a one-year period from April 2005 to April 2006. All requests for hormonal assays performed in the laboratory for hyperthyroidism, regardless of its etiology, were considered. thyrotoxicosis, or any other reason that may lead to the diagnosis of hyperthyroidism. One thousand four hundred and sixty (1460) patients were enrolled in the study, of whom $1293(89 \%)$ were sex ratio 7, 74 . The average age was 39 years with extremes 1 and 78 years. The 21-45 age group was the most represented with 27 cases. The origin of the patients referred for hormonal dosages was from all the geographical areas of Senegal with a predominance of the Dakar region 93\%; In Dakar the requests came mainly from $\mathrm{CHU}$ Abass NDAO, Dantec and FANN which recorded respectively: $41 \% ; 19.7 \%$ and $7.8 \%$. The most common hormonal dosage indication was control of antithyroid drug therapy (ADT). 596 cases (40.8\%) followed by "balance sheet" and goiter with respectively $14.6 \%$ and $12.7 \%$; TSHus was requested in $90 \%$ of patients, FT4 $81 \%$ and FT3 41\%. The TSHus demand combined with FT4 and FT3 was noted for diagnosis in 680 cases $(46 \%)$ and for the control of ADT treatment in 170 cases $(25 \%)$. The biological diagnoses were euthyroid $39 \%$, hyperthyroidism $30.80 \%$, hyperthyroidism frustrated $3.4 \%$. A discrepancy in the biological assessment was noted in 25 cases $(1.7 \%)$. From these results we find that a lot of demand is not justified. These preliminary results may be expanded by other studies in this direction. The diagnosis of right hyperthyroidism responds to a rigorous parsimony in the use of available means whose cost is a real limiting factor for the subject. 


\section{Introduction}

Biological assays for thyroid hormones are commonly used in medicine and are widely prescribed. All doctors, specialists or generalists, are required to prescribe thyroid biological tests. Indeed, in addition to clinical evaluation, biological exploration of the thyroid helps the clinician confirm the balance or imbalance of thyroid function. Thus, the reason for the request for a clear assessment can help the biologist validate the results. Inappropriate use of biological assessments can lead to unnecessary demands for testing and high health costs. Although several tests for thyroid function are available, thyroid stimulating hormone (TSH) is recommended as a first-line test for diagnosis and monitoring thyroid dysfunction. This state of play motivated this study with the aim of studying the frequency and evaluating the prescription of a thyroid test at the biophysics laboratory of Dakar.

\section{Materials and Methods}

\section{Material}

Benchmark register of the year 2015

Calculator

White paper

Computer

Pen

SPSS STATISTICS 18.0 software

\section{Methodology}

This is a descriptive cross-sectional retrospective study conducted in September 2017 for the period from January 1 to December 31, 2015. The methodology adopted is the counting of the register and the collection of the number of patients who have had a thyroid assessment in their breast. of the laboratory of biophysics of the University Cheikh Anta Diop of Dakar with as diagnosis a hyperthyroidism and results of dosage obtained. A data collection sheet has been set up to facilitate collection with the following indicators:

Sociodemographic characteristics: sex, age grouped by slice, provenance; the address of the patients.

The hormonal balance required: Serum concentration of free triiodothyronine (FT3), free tetraiodothyronine (FT4), and hypersensitive pituitary thyroid stimulating hormone (TSH us).

Clinical data: the diagnosis chosen, the reason for the assessment.

Finally this information will be entered and exploited thanks to SPSS STATISTICS 18.0 software

\section{Results and Discussion}

\section{Framework of study}

The study was carried out at the biophysics laboratory of the Faculty of Medicine of the Cheikh Anta Diop University of Dakar located on Cheikh Anta Diop Avenue, the Corniche Road and the Canal IV Boulevard. The biophysics laboratory is reputed to be the reference in terms of hormonal assessment of dysthyroidies.

\section{Criteria for inclusion and non-inclusion}

All hyperthyroid patients with a complete or partial thyroid hormone test (TSHus, FT4, FT4 + TSHus, FT3 + FT4, FT3 + TSHus, FT3 + FT4 + TSHus) were included in this study.

Not included were subjects whose names were on the register and who did not have a hormonal checkup and patients sent for hypothyroidism. 


\section{Study population}

Our study population is made up of 1460 patients, including 1293 women $(88.6 \%)$ and 167 men (11.4\%), hence a sex ratio of $7.7 \%$ for women.

\section{Distribution of study population by age}

The average age was 39 years old with extremes ranging from 1 to 78 years old. The age group (21 - 45 years) was the most represented at $64.6 \%$ and the over $60 \mathrm{~s}$ accounted for $2.2 \%$.

\section{Distribution of study population by provenance}

The patients came from Abass Ndao hospital for $41.95 \%$ of cases and from Aristide A. Le Dantec hospital accounted for $19.7 \%$.

The geographic origin of patients was dominated by the Dakar region for $92 \%$ of cases.

\section{Distribution of the study population according to the indication}

According to Table 1, the most frequent indication was the control of the treatment found in 596 patients, or $40.8 \%$ of the cases.

The indication posed as "balance sheet" was represented in 213 cases, or $14.6 \%$. Isolated or pregnancy-related disease was represented in 144 cases, or $9.9 \%$ of cases. Thyroid nodule was represented in our series in $2 \%$ of cases. The thyroid nodule plus thyrotoxicosis was represented in 14 cases (1\%) and consisted of 2 men and 12 women.

Goiter isolated or associated with thyrotoxicosis was represented in 20\% of cases. The indication of Graves' disease was represented in 135 cases, or $9.2 \%$
Distribution of the study population according to the requested balance sheet

According to Table 2, TSHus was requested in $90 \%$. FT4 in $81 \%$ and FT3 in $49 \%$. The demanded simultaneously FT3, FT4 and the TSHus was noted in 680 cases is $46.6 \%$. The simultaneous demand for FT3 and FT4 was 49\%. The dosage application of FT3 and TSHus simultaneously without FT4 was represented in $0.3 \%$. The simultaneous demand of FT4, TSHus without FT3 was represented in $25 \%$ of cases. FT4 demand alone accounted for $7.5 \%$ of cases. The demand for TSHus alone was found in $18.3 \%$.

Distribution of the study population according to the assessment requested according to the indications

According to Table 3, according to the clinical diagnosis the demand of FT3, FT4, TSHus simultaneous was the most recorded in the indications: therapeutic control (170 cases or 28.52\%) and "balance sheet": 132 cases. In the thyroid nodule isolated or associated with a thyrotoxicosis the demand for FT3, FT4, TSHus was 26 cases or $3.8 \%$. The FT3 was asked the most on the indications of the therapeutic control (72 cases) and the balance sheet (144 cases). The FT4 application for treatment control accounted for 429 cases, or $72 \%$. The demand for TSHus was strongly represented in the indications "control treatment" in 513 cases (32.2\%), and balance sheet (198 cases). Hyperthyroidism was diagnosed by the determination of TSHus alone in $25 \%$ of cases. The demand for TSHus in the diagnosis of the thyroid nodule was objectified in 3 cases, or $1 \%$. The demand for FT4 in the thyroid nodule indication was represented by 1 case (1\%). The demand for TSHus alone without FT3, FT4 in the diagnosis of thyroid nodule + thyrotoxicosis was zero (0\%) and TSH was required in $27 \%$ of cases. 
Table.1 Distribution of patients according to indications

\begin{tabular}{|l|l|l|}
\hline Indications & effective & Percentage \% \\
\hline control treatment & 596 & 40,8 \\
\hline Goiter & 185 & 12,7 \\
\hline Basedow (Graves' disease) & 135 & 9,2 \\
\hline Hyperthyroidism & 80 & 5,5 \\
\hline Thyrotoxicosis & 64 & 4,4 \\
\hline Goiter and Thyrotoxicosis & 45 & 3,1 \\
\hline Thyroidectomy & 37 & 2,5 \\
\hline Thyroid nodule & 32 & 2,2 \\
\hline Nodule and Thyrotoxicosis & 14 & 1 \\
\hline Palpitations & 15 & 1 \\
\hline Basedow and pregnancy & 9 & 0,6 \\
\hline exophthalmia & 8 & 0,5 \\
\hline GHMN & 8 & 0,5 \\
\hline GOITER BASEDOWFIE & 4 & 0,3 \\
\hline Cardiothyreosis & 5 & 0,3 \\
\hline Pre-anesthetic visit & 5 & 0,3 \\
\hline HTA & 3 & 0,2 \\
\hline balance sheet & 215 & 14,7 \\
\hline Total & 1460 & 100 \\
\hline
\end{tabular}

Table.2 Distribution of patients according to the requested balance sheet

\begin{tabular}{|c|}
\hline Balance sheet requested \\
\hline TSH \\
\hline FT3 FT4LTSH us \\
\hline FT3FT4 \\
\hline TSHusFT4 \\
\hline TSHusFT3 \\
\hline FT4 \\
\hline FT3 \\
\hline
\end{tabular}

\begin{tabular}{|c|}
\hline Number \\
\hline 1315 \\
\hline $\mathbf{6 8 0}$ \\
\hline 32 \\
\hline $\mathbf{3 6 5}$ \\
\hline $\mathbf{5}$ \\
\hline $\mathbf{1 1 6 8}$ \\
\hline 716 \\
\hline
\end{tabular}

\begin{tabular}{|c|}
\hline Percentage $\%$ \\
\hline $\mathbf{9 0}$ \\
\hline $\mathbf{4 7}$ \\
\hline $\mathbf{2 , 2}$ \\
\hline $\mathbf{2 5}$ \\
\hline $\mathbf{0 , 3}$ \\
\hline $\mathbf{8 1}$ \\
\hline 49 \\
\hline
\end{tabular}

Table.3 Distribution of patients according to the requested balance sheet According to the indications

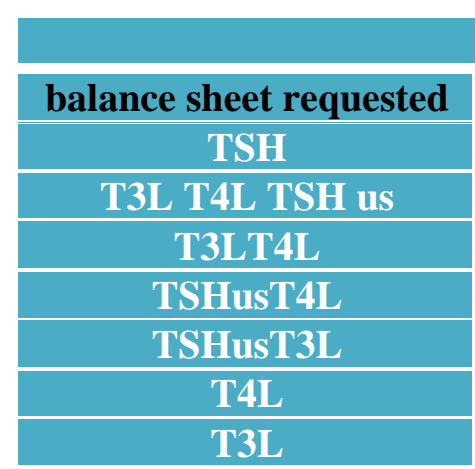

\section{INDICATIONS}

\begin{tabular}{|c|c|c|c|}
\hline control treatment & Basedow & Thyroid Nodule & Hyperthyroidism \\
\hline $27 \%$ & & $11,53 \%$ & $25 \%$ \\
\hline $29 \%$ & & & $5 \%$ \\
\hline $6 \%$ & $2,96 \%$ & & \\
\hline & & & \\
\hline $72 \%$ & $0,74 \%$ & & $1 \%$ \\
\hline $28,85 \%$ & $87,40 \%$ & $51,17 \%$ & $61,25 \%$ \\
\hline
\end{tabular}




\section{Distribution of the study population} according to biological results

The indication of Graves'disease accounted for $53 \%$ of biological hyperthyroidism.

The crude hyperthyroidism was represented in 13 cases $(9.6 \%)$. The peak frequency was represented by hyperthyroidism with $31 \%$. Euthyroid accounted for 163 cases, or $27.3 \%$. Hyperthyroidism partially compensated, accounted for 117 cases or $19.6 \%$. Iatrogenic hypothyroidism was noted in 86 cases, or $14.4 \%$. Euthyroid dominated the biological diagnosis of the thyroid nodule in 23 cases or $88.46 \%$. The biological diagnosis of the thyroid nodule and thyrotoxicosis was dominated by euthyroid for 8 cases or $57.1 \%$ followed by hyperthyroidism in 3 cases. The balance was disrupted in 25 cases or $1.7 \%$.

This study allowed us, thanks to the good behavior of the register of the laboratory of biophysics and dosing the thyroid hormones, to know the profile of the prescription of the assays of the thyroid balance. In fact, the assays of the thyroid hormones and the TSHus are prescribed for recognition and monitoring of abnormalities of thyroid function. Our approach was to evaluate in hyperthyroidism the elements that motivated the request for a biological assessment according to the diagnostic suspicion with an analytical approach to reduce the number and therefore the cost of dosages while remaining effective on the management. At the end of this study, we recorded 1460 assays for the exploration of hyperthyroidism at the biophysics laboratory in Dakar. The average age is 39 years which is like that of (Monabeka, 2005) who finds an average age of 34 , with extremes of 18 and 71 years. In our study the TSHus was requested in $90 \%$, the FT4 in $81 \%$ and the FT3 in 49\%. For (Monabeka, 2005) FT3 and TSHus were performed in $24(20.3 \%)$ and 69 patients
(58.5\%), A survey conducted in France shows that highly sensitive TSH is the most performed dosage (43\% of prescriptions), but T3 (FT3 or TT3) is frequently prescribed (22\%) (Vidal-Trecan, 1999). Several studies show that most thyroid dysfunctions, whether patients are hospitalized or not, are identified or excluded by a TSHus assay possibly associated with that of FT4 (Davey et al., 1996; Nordyke et al., 1998). Second- and third-generation TSHus assays detect subclinical hypo- or hyperthyroidism and allow treatment follow-up; the determination of FT4 allows the diagnosis of central hypothyroidism and better detects rapid changes in thyroid function (Nordyke et al., 1998). The follow-up of a decision algorithm, with first-line isolated TSHus assay, is recommended by some authors (Feldkamp and Carey, 1996). However, for a correct assessment of the thyroid function, the American Thyroid Association recommends the coupling of the TSHus assays and FT4 (Singer et al., 1995). The adequacy of biological tests in medical practice is a fundamental problem, because it is a question of optimally managing patients while controlling health expenditure (We know what a laboratory is a systematic review of laboratory clinical audits, 1998). Thanks to the awareness of the concept of costeffectiveness, limiting unnecessary additional examinations and / or not complying with indications, often due to major technical progress, has become a topical concern. To optimize medical practice, algorithms and / or recommendations for the prescription of thyroid status have been published (Becker $e t$ al., 1993). Information and harmonization of practitioners is certainly key to improving the way they prescribe. (Wemeau and D'herbomez-Boidein, 2005) The analysis of the prescription of thyroid hormones has already been the subject of many publications, especially since the advent of third generation TSHus assays, the aim being to restrict the 
inappropriate prescriptions mainly of FT3 (free triiodothyronine) and / or FT4 (free thyroxine) (Bauer and Brown, 1996)

In Senegal the dosage of thyroid hormones is not reimbursed. In our series, therapeutic control showed 28.5\%, euthyroid and hyperthyroidism in $31 \%$ of cases. This hyperthyroidism despite treatment with ATS may be due to a relapse or a therapeutic failure. (Torring et al., 1996) found in a prospective study a $38 \%$ relapse rate. The therapeutic failure may be due to poor compliance due to a long and expensive treatment. Therapeutic education is necessary to ensure effective treatment. Hyperthyroidism accounts for $3.4 \%$ in our series. This agrees with the literature which describes a prevalence of 0.7 to $3.9 \%$ (Bahn et al., 2011). In our study the balance was disrupted in 25 cases or $1.7 \%$. For (Monabeka, 2005) There were discrepancies between TSHus and FT4 in 11 patients, or 9.32\% (Monabeka, 2005). When there is discrepancy, a dialogue between the clinician and the biologist is needed to discuss the potential causes. It is important for this purpose to clearly elucidate the indications that motivated the request of the biological assessment to help the biologist to validate the results. The balance pattern was noted in $14.6 \%$ of patients. Our study shows that, as it stands, the increase in the number of noncompliant practices in terms of balance sheet prescription remains well established, detrimental to patients.It is up to us to remind the various prescribers of certain rules in line with the new recommendations to overcome these insufficiencies or systematic prescriptions of triple dosages. Other studies of the same nature could confirm or invalidate these first results which call for a rationalization of thyroid assessment

This study shows the importance of rationalization in the prescription of the thyroid hormone balance. This study showed an increase in the systematization of the dosage of FT3 + FT4 + TSHus in phase with the recommendations to that effect. It is therefore important to make the diagnosis well to reduce the non-compliant practices, thus conditioning the parameter to be requested and thus improving the patients' stock market for a respect of the balance sheets and an adequate observance of the treatment.

\section{References}

Bahn RS, Burch HB, Cooper DS, et al., Hyperthyroidism and other causes of thyrotoxicosis: management guidelines of the American Thyroid Association and American Association of Clinical Endocrinologists. Thyroid 2011; 21: 593-646

Bauer DC and Brown AN. Sensitive thyrotropin and free thyroxine testing in outpatients. Arch Intern Med 1996; 11: 2333-2337

Becker DV, Bigos ST, Gaitan E, Morris JC, R and amp; ML, Spencer CA, Sugawara M, Van Middlesworth L, Wartofsky L. Optimal use of blood tests for assessment of thyroid function. JAMA 1993; 269: 2736-2737

Davey RX, MI Clarke, Webster AR. Thyroid function testing based on thyroidstimulating hormone: assessing an algorithm's reliability. MJA 1996; 164: 329-32.

Feldkamp CS, and Carey JL. An algorithmic approach to your testing in a managed care setting. Am J ClinPathol 1996; 105: 11-6.

Georges P. Thyroid hormonal assays Public Assistance - Paris hospitals: prescriptions, cotit and opinions of the patients qualified in endocrinology. Ann Emkxrin 1999; 60: 48-55 Zaat OJ, van Eijk JT, Bonte HA. Laboratory test 
form

Monabeka, H.G. Management of hyperthyroidism at the University Hospital of Brazzaville, Congo. Bull. Soc.Pathol.Exot., 2005, 98, 2, 91-93

Nordyke, R.A., Thomas TS, LD Madanay, JC Woods, Goldstein AP, Miyamoto LA. Alternative sequences of thyrotropin and thyroxin free assays for routine thyroid function testing. Quality and cost. Arch Intern Med 1998; 158: 26672.

Singer PA, Cooper DS, Levy EG et al., Treatment guidelines for patients with hyperthyroidism and hypothyroidism. JAMA 1995; 273: 808-12.

Torring O, Tallstedt L, Wallin G, Lundell G,
Ljunggren JG, Taube A, et al., Graves' hyperthyroidism: treatment with antithyroid drugs, surgery, or radioiodine. A prospective randomized study. J Clin Endocrinol Metab 1996; 81:(8) 2986- 93.

Vidal-Trecan, G. Thyroid hormone assays in Public Assistance - Annecy Hospitals of Paris Endocrinology Vol 60, $\mathrm{N}^{\circ} 1$ May 1999 p. 48

We know what a laboratory is a systematic review of laboratory clinical audits. JAM4 1998; 280: 550-558

Wemeau JL, D'herbomez-Boidein M. Biological exploration of the thyroid: what examinations, for what indications? Biotribune, 2005; 14: 30

\section{How to cite this article:}

Djiby Sow, Amadou Ndiaye, Boubacar Ndong, Moussa Badiane, Pr Oumar Ndoye and Pr Niama Diop Sall. 2018. Hormonal Balance of Hyperthyroidism in the Laboratory of Biophysics of Dakar: Proposal of a Diagnostic and Monitoring Approach. Int.J.Curr.Microbiol.App.Sci. 7(01): 3466-3472. doi: https://doi.org/10.20546/ijcmas.2018.701.408 